\title{
Sudden Unexpected Death in Epilepsy
}

Rathnaweera Hewawitharanage Ajith Indika Rathnaweera* $\triangle(D$

Department of Forensic Medicine, Faculty of Medicine, Karapitiya, Galle, Sri Lanka.

\section{Introduction}

Patients with epilepsy have a mortality rate significantly higher than that of the general population. Sudden unexpected death in epilepsy is a major diagnostic category in epilepsy, accounting for between 500 and 1000 deaths per year.

\section{Case reports}

Case 1: A 65-year-old male with a past history of epilepsy had a witnessed seizure. Approximately two hours later he was found unresponsive. No significant natural diseases were found at the autopsy. Neuropathological examination showed non-specific changes within the hippocampus. Toxicological analysis detected carbamazepine and valproic acid. Vitreous humour biochemistry was unremarkable. Case 2: A 56-year-old male with a past history of epilepsy was found by his mother unresponsive. Resuscitation was attempted but failed. No significant natural diseases were found at the autopsy. The neuropathological examination did not reveal an epileptic focus. Toxicological analysis revealed carbamazepine and phenytoin. Vitreous humour biochemistry was unremarkable. Case 3: A 45-year-old female with a past history of epilepsy was found on the floor unresponsive. She was off antiepileptic treatment for the last two weeks. No significant natural diseases were found at the autopsy. The neuropathological examination did not reveal an epileptic focus. The regular antiepileptic medications were not detected in the toxicological analysis. Vitreous humour biochemistry was unremarkable.

\section{Conclusions}

The above-mentioned cases were classical SUDEP deaths. The diagnosis of SUDEP mainly depends on exclusion. The need of thorough histopathology examination including neuropathology, a complete toxicological analysis including vitreous humour biochemistry is highlighted here in the event of epileptic deaths.

Keywords: Epilepsy, SUDEP, Hippocampal sclerosis, Neuropathology

Copyright: @ 2017 with the Medico-legal Journal of Sri Lanka.

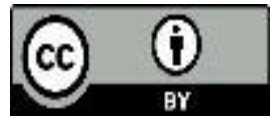

This is open access article distributed under the terms of the Creative Commons Attribution License, CC BY 4.0 which permits unrestricted use, distribution and reproduction in any medium, provided the original author and source are credited.

Funding: None, Competing interests: None

Received: 14 November $2017 \quad$ Accepted revised version: 20 March 2018 Published: 01 April 2018

http://orcid.org/0000-0002-7418-2981

*Corresponding author: Rathnaweera RHAI, Email address: ajithrathnaweera@ gmail.com $\triangle$, Tel: +94772969060 Cite this article as: : Rathnaweera RHAI. Sudden Unexpected Death in Epilepsy. Medico-Legal Journal of Sri Lanka, 2017;5(2):26-28

DOI: http://dx.doi.org/10.4038/mljsl.v5i2.7361 


\section{Introduction}

Mortality due to epilepsy is a significant concern. Patients with epilepsy have a mortality rate significantly higher than that of the general population. ${ }^{[1]}$ This excess is made up of a number of components, including increased incidence of neoplasm, seizure-related death (accidents, asphyxia/aspiration, status epilepticus), and sudden unexpected death. ${ }^{[2]}$ Sudden unexpected death in epilepsy (SUDEP) is a major diagnostic category in epilepsy, accounting for between 500 and 1000 deaths per year in England. ${ }^{[3]}$

Case 1: A 65-year-old male with a past history of epilepsy was found unresponsive two hours after a witnessed seizure. Resuscitation was attempted but failed. The last documented seizure was about 2 years prior to this event and his epilepsy had been well controlled, according to his local medical practitioner. His regular medications included carbamazepine and sodium valproate. The postmortem examination did not reveal any significant natural illness. Neuropathological examination showed non-specific changes within the hippocampus and cerebellar vermis. No epileptic focus was identified. Toxicological analysis detected carbamazepine and valproic acid, the deceased's prescribed medications for epilepsy, indicating that the deceased was taking these medications prior to death. Vitreous humour biochemistry showed normal levels of urea and electrolytes while vitreous glucose was undetectable. The cause of death was given as sudden unexpected death in epilepsy.

Case 2: A 56- year-old male was found unresponsive by his mother. Resuscitation was attempted but failed. The deceased had a history of epilepsy and the last seizure episode occurred around one year ago. Postmortem examination did not reveal any natural disease condition. Neuropathological examination was unremarkable. Toxicological analysis revealed carbamazepine and phenytoin. Although carbamazepine was within the normal therapeutic range for treatment of epilepsy, phenytoin was below the therapeutic range. This could be either due to postmortem redistribution of the drug or subtherapeutic concentrations. Vitreous humour biochemistry showed normal levels of urea and electrolytes while vitreous glucose was undetectable. The cause of death was given as sudden unexpected death in epilepsy.

Case 3: A 45- year-old female with a past history of right temporal lobe epilepsy was found on the floor unresponsive by her ex-husband. She was off antiepileptic treatment for the last two weeks. Resuscitation was attempted but failed. The natural disease identified at postmortem (myocardial fibrosis and borderline cardiomegaly) was considered not sufficient to cause death in this case. The neuropathological examination did not reveal an epileptic focus. The regular antiepileptic medications were not detected in the toxicological analysis. Vitreous humour biochemistry showed normal levels of urea and electrolytes while vitreous glucose was undetectable. The cause of death was given as sudden unexpected death in epilepsy.

\section{Discussion}

SUDEP is defined as 'sudden, unexpected, witnessed or unwitnessed, non-traumatic and non-drowning deaths in a patient with epilepsy, with or without evidence of a seizure and excluding documented status epilepticus, in which post-mortem examination does not reveal a toxicological or anatomical cause of death. $^{[4]}$

The mechanism of death in these cases is not clearly understood. There are indications that cardiac mechanisms play a role, due to repetitive cardiac damage during seizures and due to arrhythmia during seizures, possibly triggered by apnea and/or cerebral autonomic imbalance. Furthermore, undetected cardiac or respiratory diseases predisposing for arrhythmias or apnea may play a role in SUDEP. ${ }^{[5]}$

Diagnosis of SUDEP is mainly done by exclusion. Initial information should include the certainty of the clinical diagnosis of epilepsy during life. Anatomical and toxicological cause of death exclusion plays a major part in the diagnosis. Out of the anatomical causes, detection or exclusion of a cardiac disease as the cause of death may be difficult even at autopsy. A thorough histopathological examination of the heart muscle is required to detect pathologic substrates like focal myocarditis, hypertrophic or dilative cardiomyopathy, arrhythmogenic right ventricular dysplasia or diseases of the conduction system. However, defects in the ion channels or pre -excitation syndromes, like long QT syndrome, Brugada syndrome or WPW-syndrome may lead to sudden cardiac death with no evidence of cardiac pathologies at the macroscopic or microscopic investigation. ${ }^{[6]}$

By definition, in SUDEP deaths, significant swelling, shift or herniation of brain is absent. Hippocampal sclerosis is the most common lesional pathology identified in patients with temporal lobe epilepsy and has been reported in patients with SUDEP. Amygdala 
sclerosis also has been reported in some SUDEP cases. Specific patterns of neuronal loss may have functional implications through a susceptibility to cardiac arrhythmias and act as a risk factor for SUDEP. ${ }^{[7]}$

The difficulty in diagnosis of SUDEP lies mainly in its thorough investigation process. A comprehensive histopathology examination of all organs including neuropathological analysis of brain is essential in arriving at a final diagnosis. A comprehensive toxicological analysis including therapeutic drug levels and vitreous humour biochemistry is also very important in the exclusion process. Even though SUDEP remains a common cause of death in developed countries, it is a very rare diagnosis in developing countries. The main reason for this is the inability to do a proper exclusion owing to the fact of lack of resources. Because of this, the final diagnosis may remain as unascertained in potential SUDEP cases.

All the discussed cases in this paper were potential SUDEP deaths with a classic past history, a seizure episode followed by sudden and unexpected death. In case 1 , the deceased had the previous seizure about two years ago and was on regular treatment.
In case 2 , the deceased had the previous seizure about one year ago and in case 3 , the exact date of previous seizure was not known as she was not on regular medication for the past two weeks. The toxicological analysis was in keeping with the medication history of all three cases. As proper and standard investigations were carried out in all these cases, a proper exclusion of other causes of deaths could have been done and the final diagnosis of SUDEP was made.

\section{Conclusions}

The above mentioned cases were classical SUDEP deaths. The diagnosis of SUDEP was made with proper exclusion. The need of thorough

histopathology examination including neuropathology, a complete toxicological analysis including vitreous humour biochemistry is highlighted here in the event of epileptic deaths. A systematic improvement of facilities in the forensic laboratories is vital in the management of epileptic deaths so that a proper exclusion can be made with the view of making the correct diagnosis of SUDEP.

\section{References}

1. Klenerman P, Sander J, Shorvon D. Mortality in patients with epilepsy: a study of patients in long-term residential care. Journal of Neurology, Neurosurgery, and Psychiatry 1993;56(1):149-52. Available from: https://www.ncbi.nlm.nih.gov/pmc/articles/PMC1014813

2. Timmings L. Sudden unexpected death in epilepsy: a local audit. Seizure 1993; 2(1):287-90. Available from: https://www.ncbi.nlm.nih.gov/m/pubmed/8162397

3. Brown W, Mawer E, Lawler E, Taylor C. Sudden death and epilepsy. Lancet 1990; 335(1): 606- 7. Available from:

https://www.ncbi.nlm.nih.gov/pmc/articles/PMC1293687

4. Nashef L, Shorvon D. Mortality in epilepsy. Epilepsia 1997;38(1):1059-

61. DOI: https://doi.org/10.1111/j.1528-1157.1997.tb01193.x

5. Ryvlin P, Montavont A, Kahane P. Sudden unexpected death in epilepsy: from mechanism to prevention. Curr opin Neurol 2006;19(1): 194-9.

DOI: http://doi.org/10.1097/01.wco.0000218238.90711.f4

6. Thom M. The autopsy in sudden unexpected adult death: Epilepsy. Current diagnosis pathology 2007;13(1): 389-400.

DOI: http://doi.org/10.1016/j.cdip.2007.05.004

7. Black M, Graham I. Sudden unexpected death in adults caused by intracranial pathology. J clin pathol 2002;55(2): 44-50. 